\title{
Estimation in a fluctuating medium and power-law distributions
}

\author{
C. Vignat ${ }^{\mathrm{a}, *}$, A. Plastino ${ }^{\mathrm{b}}$ \\ ${ }^{a}$ LPM, EPFL, Lausanne, Switzerland \\ ${ }^{\mathrm{b}}$ La Plata National University, Exact Sciences Faculty \& National Research Council (CONICET), C.C. 727, 1900 La Plata, Argentina
}

Received 7 June 2006; accepted 3 July 2006

Available online 14 July 2006

Communicated by A.R. Bishop

\begin{abstract}
We show how recent results by Bening and Korolev in the context of estimation, when linked with a classical result of Fisher concerning the negative binomial distribution, can be used to explain the ubiquity of power-law probability distributions. Beck, Cohen and others have provided plausible mechanisms explaining how power-law probability distributions naturally emerge in scenarios characterized by either finite dimension or fluctuation effects. This Letter tries to further contribute to such an idea. As an application, a new and multivariate version of the central limit theorem is obtained that provides a convenient alternative to the one recently presented in [S. Umarov, C. Tsallis, S. Steinberg, cond-mat/0603593]. (C) 2006 Elsevier B.V. All rights reserved.
\end{abstract}

PACS: 05.30.-d; 05.30.Jp

\section{Estimation in a fluctuating context}

Beck, Cohen, and others have provided strong indications concerning the way in which power law probability distributions (PDs) naturally emerge in scenarios characterized by either finite dimension or fluctuation effects [1-7]. In a parallel vein, we wish here to offer a purely statistical argument to the same effect, that clearly exhibits a simple mechanism that operates so as to lead to the appearance of these PDs. This mechanism is based on a more general result issued from estimation theory $[8,9]$.

In the conventional estimation scenario, a series of independent random data $\left\{X_{i}\right\}$ is observed; their common distribution $P_{\theta_{0}}$ is supposed to belong to a parameterized set of distributions $\left\{P_{\theta} ; \theta \in \Theta\right\}$. A statistics $T_{n}\left(X_{1}, \ldots, X_{n}\right)$ is defined as a measurable function of the observed data. This statistics is called asymptotically normal if it verifies the following property: there exist functions $\delta(\theta)$ and $t(\theta)$ such that the distribution

$$
\mathbf{P}_{\theta}\left\{\delta(\theta) \sqrt{n}\left(T_{n}-t(\theta)\right)<x\right\}
$$

\footnotetext{
* Corresponding author.

E-mail address: christophe.vignat@epfl.ch (C. Vignat).
}

converges weakly to the normal distribution as $n \rightarrow+\infty$. Normal statistics are ubiquitous in the real world, sample mean and maximum likelihood estimators being notable examples.

Before proceeding we recall that

- the gamma distribution function with scale parameter $\alpha$ and shape parameter $\lambda$ is defined as

$$
G_{\alpha, \lambda}(x)= \begin{cases}\int_{0}^{x} \frac{\lambda^{\alpha}}{\Gamma(\alpha)} e^{-\lambda y} y^{\alpha-1} d y & \text { if } x \geqslant 0, \\ 0 & \text { else; }\end{cases}
$$

- the $d$-variate t-distribution or Student's t-distribution with $\gamma$ degrees of freedom $F_{\gamma}\left(x_{1}, \ldots, x_{d}\right)$ is a probability distribution that arises in the problem of estimating the mean of a normally distributed population when the sample size is small. It writes ( $x$ and $y$ below are $d$-dimensional vectors and the superscript $t$ denotes transposition)

$$
\begin{aligned}
& F_{\gamma}\left(x_{1}, \ldots, x_{d}\right) \\
& =\frac{\Gamma\left(\frac{\gamma+d}{2}\right)}{(\pi \gamma)^{d / 2} \Gamma\left(\frac{\gamma}{2}\right)} \\
& \quad \times \int_{-\infty}^{x_{1}} \cdots \int_{-\infty}^{x_{d}}\left(1+\frac{y^{t} y}{\gamma}\right)^{-\frac{\gamma+d}{2}} d y_{1} \cdots d y_{d} .
\end{aligned}
$$


Assume now, and this is our critical point here, that a random number of data is available to build statistics $T$. This is in fact often the case in real physical experiments such as multiparticle detection [10-12] or photon statistics [13-16]. One assumes that there exists a family of integer valued random variables $\left\{N_{n}\right\}$ that are independent of the observed data $\left\{X_{i}\right\}$. We say that $N_{n} \rightarrow \infty$ in probability as $n \rightarrow \infty$ if, $\forall K>0$

$\lim _{n \rightarrow+\infty} \operatorname{Pr}\left\{N_{n} \geqslant K\right\}=1$.

In such a situation, a notable result of Bening and Korolev's becomes applicable [17, Theorem 2.1]; we give here an immediate multivariate version of this result as specified by the following

Theorem 1. Let $\gamma>0$ be arbitrary and let $\left\{d_{n}\right\}_{n} \geqslant 1$ be some infinitely increasing sequence of positive numbers. Suppose that $N_{n} \rightarrow \infty$ in probability as $n \rightarrow \infty$ with respect to any probability from a family $\left\{P_{\theta} ; \theta \in \Theta\right\}$. Let the statistic $T_{n} \in \mathbb{R}^{p}$ be asymptotically normal. In order to have, for any $\theta \in \Theta$,

$\mathbf{P}_{\theta}\left\{\delta(\theta) \sqrt{d_{n}}\left(T_{N_{n}}-t(\theta)\right)<x\right\} \Rightarrow F_{\gamma}(x), \quad n \rightarrow \infty$,

where $F_{\gamma}(x)$ is the multivariate Student $t$-distribution function with $\gamma$ degrees of freedom, it is necessary and sufficient that for any $\theta \in \Theta$,

$\mathbf{P}_{\theta}\left\{N_{n}<d_{n} x\right\} \Rightarrow G_{\gamma / 2, \gamma / 2}(x), \quad n \rightarrow \infty$.

The proof is immediate from the univariate version in [17, Theorem 2.1] and relies on the stochastic representation of a multivariate t-distributed random vector $X$ with $\gamma$ degrees of freedom as the Gaussian mixture [18,19]

$X=\frac{G}{\sqrt{a}}$

where $G$ is a multivariate Gaussian vector and $a$ is a scalar random variable independent of $G$ following a gamma distribution with shape parameter $\gamma / 2$.

As an example of a family of random number of data $\left\{N_{n}\right\}$ that verifies condition (5), Bening and Korolev provide the negative binomial (or Pascal) distribution: given a positive real $r$ and a probability $0<p<1$, this distribution is

$\operatorname{Pr}\{N=k\}=\left(\begin{array}{c}k \\ r+k-1\end{array}\right) p^{r}(1-p)^{k}, \quad k=0,1, \ldots$

Note that this distribution was independently characterized in [12] as the particles multiplicity distribution that ensures that the energies associated with these particles follow a $q$ exponential distribution. In order to give an interpretation for $N$, recall at this point the Bernoulli process, one of the simplest yet most important random processes in probability. Essentially, it is the mathematical expression of coin tossing, but because of its wide applicability, it is usually stated in terms of a sequence of generic trials that satisfy the following assumptions: (i) Each trial has two possible outcomes, generically called success and failure. (ii) The trials are independent (the outcome of one trial has no influence over the outcome of another one). (iii) On each trial, the probability of success is $p$ and the probability of failure is $1-p$. If in Eq. (7) above we assume that $r \in \mathbb{N}$, then $N$ can indeed be interpreted, in a series of Bernoulli trials, as the number of failures necessary to obtain a final $N$ th success after $r-1$ successes. If $p$ is chosen according to

$p=\frac{1}{n}$,

then convergence as in (4) holds with $d_{n}=r n$ and $\gamma=2 r$.

The connection with power law probability distributions becomes now immediate. We only have to remember [20-23] that the $q$-Gaussian distributions $e_{q}\left(x^{t} x\right)$ with

$e_{q}(x)=[1+(1-q) x]^{1 /(1-q)}, \quad x \in \mathbb{R}^{d}, q \in \mathbb{R}$,

are power-law distributions that maximize Tsallis entropy under covariance constraint $E x x^{t}=\left\langle x x^{t}\right\rangle=K$ and that, for [19]

$1<q<\frac{d+4}{d+2}$

they coincide with $d$-variate Student t-distributions with $r$ degrees of freedom, provided that

$r=\frac{2-d(q-1)}{q-1}$.

One then concludes that an asymptotically normal statistics $b e$ comes an asymptotically $q$-Gaussian statistics if it is built upon data whose number fluctuates according to a negative binomial distribution, as in (7).

\section{The negative binomial distribution}

The shape of the negative binomial distribution and its dependence on parameters $p$ and $r$ are illustrated by Figs. 1 and 2 below. In Fig. 1 the parameter $r$ is fixed to $r=5$ while parameter $p$ takes values in the set $\{1 / 10,1 / 15,1 / 20,1 / 25\}$. In Fig. 2 the parameter $p$ is fixed to $p=1 / 2$, while parameter $r$ takes values in the set $\{5,10,15,20,25\}$. The appearance of the negative binomial in this context can be justified by reference to the following idea of Fisher [25]: Let us consider a discrete random variable $P$ that follows a Poisson distribution with parameter $\lambda$ :

$P \sim P_{\lambda}(n)=\frac{\exp (-\lambda) \lambda^{n}}{n !}$.

Now let us assume that parameter $\lambda$ is itself a random variable: we quote below Fisher in verbatim fashion [25]:

"Since $\lambda$ is necessary positive, the simplest frequency distribution which allows some variation of $\lambda$ is the Eulerian distribution, familiar as that of $\chi^{2}$, in which the frequency element is

$d f=\frac{1}{(r-1) !} p^{-r} \lambda^{r-1} e^{-\lambda / p} d \lambda$.

For $\chi^{2}$, the parameter $r$ is always the half of a positive integer; in general it may be any number exceeding zero".

We thus deduce that a negative binomial distribution is nothing but a Poisson distribution whose random parameter $\lambda$ follows (itself) a gamma distribution with parameter $2 r$ : Note that 


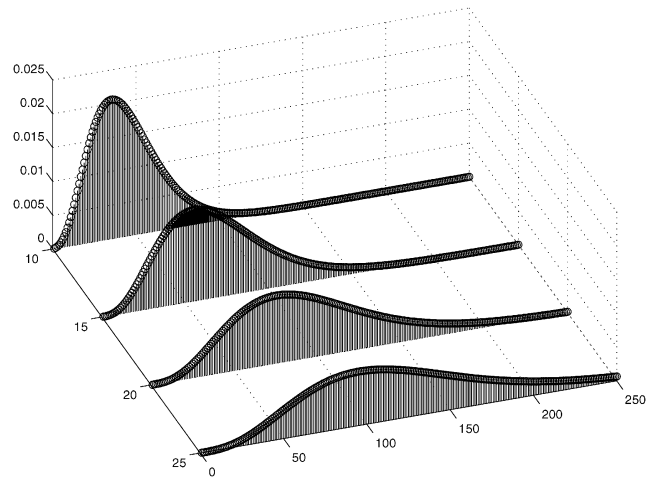

Fig. 1. The negative binomial distribution for $r=5$ and $p=1 / 10$ (back), $1 / 15$, $1 / 20$ and $1 / 25$ (front).

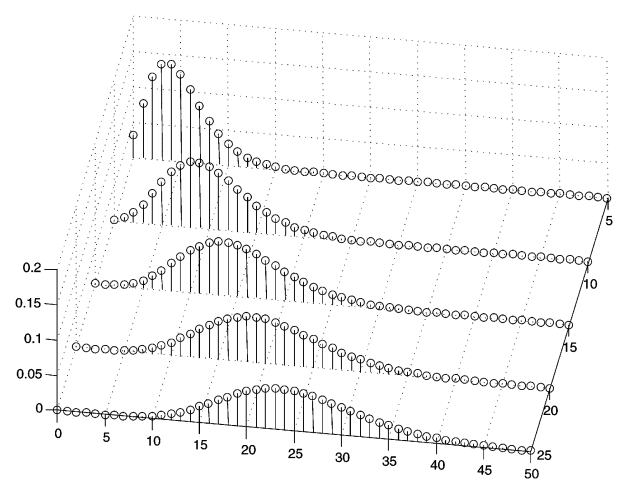

Fig. 2. The negative binomial distribution for $p=1 / 2$, and $r=5$ (back), 10, 15,20 and 25 (front).

this property is cited in both [12] and [17], but without mentioning Ref. [25].

Our central point here is the following: In a great number of experiments one observes events that are Poisson-distributed with a device that necessarily exhibits, like all instruments, positive fluctuations originated in a number of independent sources [8]. This is a fact of life that justifies the negative binomial distribution of the observed data, and a posteriori (asymptotically), the $q$-Gaussian distribution of any statistics associated to these data.

\section{Application to random summation}

As a rather important application of the above results, we explicit the following consequence, that provides us with an alternative approach to the intriguing problem of the existence of a central limit theorem for power-law distributions [24]. A classical statistics is the sample mean estimator defined as

$T_{n}=\frac{1}{n} \sum_{i=1}^{n} X_{i}$,

where $X_{i} \in \mathbb{R}^{d}, 1 \leqslant i \leqslant n$ are independent random vectors with finite covariance matrix. It is well known that this statistics is asymptotically normal. Applying now the preceding results we deduce that, if the number of data used in this statistics is itself a random variable $N_{n}$ following a negative binomial distribution with parameters $r$ and $p=1 / n$ then, under condition (8), the resulting sample mean statistics

$$
T=\frac{1}{N_{n}} \sum_{i=1}^{N_{n}} X_{i}
$$

is asymptotically (as $n \rightarrow+\infty$ ) q-Gaussian with $r$ degrees of freedom.

This result constitutes a multivariate form of the central limit theorem and represents an alternative to the recent and interesting result of Ref. [24], where a certain type of dependence between the data, called $q$-independence, is shown to ensure the asymptotic $q$-"Gaussianity" of the sample mean statistics. We underline that in the result presented here, no special type of dependence is required, since its conditions of application coincide with the conditions required in the usual central limit theorem.

\section{Conclusions}

We have shown, by applying results of Bening and Korolev [17], that $q$-Gaussian distributions necessarily emerge in the very general context of estimation theory.

More specifically, if an asymptotically normal statistics is used with a random number of data that follows a negative binomial distribution with parameters $p=1 / n$ and $r$, then the resulting statistics is in fact $q$-Gaussian-distributed, with $r$ degrees of freedom as given by (11), parameter $q$ belonging to the range of values (10). With reference to this $q$-range, precise indications as to which is the "correct" $q$-value in a given scenario are still the subject of intense debate [21,23]. Our present results may also be construed as a rather significative contribution to such debate.

\section{References}

[1] C. Beck, E.G.D. Cohen, Physica A 322 (2003) 267.

[2] C. Beck, Contin. Mech. Thermodyn. 16 (2004) 293.

[3] C. Beck, Phys. Rev. Lett. 87 (2001) 180601.

[4] C. Beck, E.G.D. Cohen, Physica A 344 (2004) 393.

[5] C. Beck, Physica A 342 (2004) 139.

[6] C. Beck, Physica D 193 (2004) 195.

[7] H. Touchette, C. Beck, Phys. Rev. E 71 (2005) 016131.

[8] B.R. Frieden, Physics from Fisher Information, Cambridge Univ. Press, Cambridge, 1998.

[9] B.R. Frieden, Science from Fisher Information, Cambridge Univ. Press, Cambridge, 2003.

[10] A.T. Bharucha-Reid, Phys. Rev. 96 (1954) 751.

[11] A.Z. Mekjian, B.R. Schlei, D. Strottman, Phys. Rev. C 58 (1998) 3627.

[12] G. Wilk, Z. Wlodarczyk, cond-mat/0603157.

[13] S.M. Barnett, J. Mod. Opt. 10 (1998) 2201.

[14] T. Ganstog, A. Josh, R. Tanas, Quantum Opt. 6 (1994) 517.

[15] B.R. Frieden, Probability, Statistical Optics, and Data Testing, Springer, Berlin, 2001.

[16] C.W.J. Beenakker, H. Schomerus, Phys. Rev. Lett. 86 (2001) 700.

[17] V.E. Bening, V.Yu. Korolev, Theory Probab. Appl. 49 (2005) 377.

[18] C. Vignat, A. Plastino, A.R. Plastino, Nuovo Cimento B 120 (2005) 951.

[19] C. Vignat, A. Plastino, Phys. Lett. A 343 (2005) 411, and references therein;

C. Vignat, A. Plastino, Physica A 361 (2006) 139;

C. Vignat, A. Plastino, Physica A 365 (2006) 167, and references therein.

[20] S. Abe, Y. Okamoto (Eds.), Nonextensive Statistical Mechanics and Its Applications, Springer, Berlin, 2001. 
[21] M. Gell-Mann, C. Tsallis (Eds.), Nonextensive Entropy: Interdisciplinary Applications, Oxford Univ. Press, Oxford, 2004, and references therein; A.R. Plastino, A. Plastino, Phys. Lett. A 193 (1994) 140.

[22] G. Kaniadakis, M. Lissia, A. Rapisarda (Eds.), Nonextensive Statistical Mechanics and Physical Applications, Physica A (Special issue) 305 (2002), and references therein.
[23] Special issue, Europhysicsnews 36 (2005);

M. Buchanan, New Sci. 187 (2005) 34.

[24] S. Umarov, C. Tsallis, S. Steinberg, cond-mat/0603593.

[25] R.A. Fisher, Ann. Eugenics 11 (1941) 182, available at http://digital. library.adelaide.edu.au/coll/special/fisher/stat_math.html. 